\title{
0 uso da plataforma Balance Board como recurso fisioterápico em idosos
}

\author{
The Balance Board plataform used as a physiotherapy resource in elderly
}

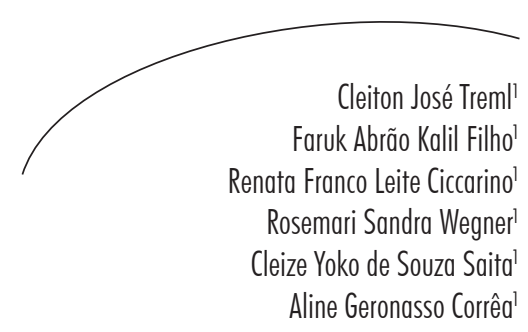

Resumo

Objetivos: Avaliar os efeitos de um programa de treinamento proprioceptivo convencional e de um protocolo com a utilização do videogame associado a Balance Board em indivíduos idosos, em relação a equilíbrio, mobilidade, flexibilidade e quedas. Método: Trata-se de pesquisa quase experimental, realizada com 32 indivíduos idosos. A amostra consistiu em dois grupos: o primeiro GC ( $\mathrm{n}=16$; idade $=67,63$ anos), com treinamento proprioceptivo convencional e o segundo, GE ( $\mathrm{n}=16$; idade $=66,88$ anos), em treinamento proprioceptivo com a nova ferramenta tecnológica. Foram realizados dez atendimentos, duas vezes na semana, duração de 30 minutos no GC com treinamento proprioceptivo convencional e 30 minutos no GE com plataforma Balance Board. Resultados: Para o GE, somente a variável escala de Berg não apresentou diferença significativa $(\mathrm{p}<0,05)$ entre os momentos pré e pós-intervenção, sendo que na escala POMA $(p=0,018)$, Unipodal $(p=0,018)$ e testes de alcance funcional anterior e lateral $(p=0,012)$ observou-se diferença estatística $(p<0,05)$ significativa nos momentos avaliados. Já para o GC, tanto POMA ( $\mathrm{p}=0,043)$ como a escala Unipodal $(\mathrm{p}=0,043)$ apresentaram diferenças significativas entre o momento pré e pós-intervenção. Conclusão: Conclui-se que o treinamento proprioceptivo com realidade virtual mostrou ser mais eficiente que o treinamento proprioceptivo convencional em indivíduos idosos em relação ao equilíbrio, mobilidade, flexibilidade e quedas.

\section{Abstract}

Objective: To evaluate the effects of a conventional proprioceptive training program and a protocol with the association of video game use and Balance Board in elderly regarding balance, mobility, flexibility and falls. Method: This almost experimental research was conducted with 32 elderly participants. The sample consisted of two groups. The first, CG ( $\mathrm{n}=16$; aged $=67.63$ years), with conventional proprioceptive training and the second, SG ( $\mathrm{n}=16$; aged $=66.88$ years) proprioceptive training with the new technological tool. Treatments were done in both groups twice a week, during

Palavras-chave:

Equilíbrio Postural. Idoso. Propriocepção.
Key words: Postural Balance. Elderly. Proprioception.

\footnotetext{
Departamento de Fisioterapia. Faculdade Evangélica do Paraná. Curitiba, PR, Brasil.
} 
30 minutes, adding up to ten interventions. Results: In SG only the Berg Scale variable did not show significant difference $(\mathrm{p}<0.05)$ between pre and post intervention. In evaluated moments significant statistic difference $(\mathrm{p}<0.05)$ was observed in the POMA ( $\mathrm{p}=0.018)$ and One-leg scale $(\mathrm{p}=0.018$, as well as in Anterior and Lateral Functional Reach tests $(\mathrm{p}=0.012)$. As for CG, both POMA $(\mathrm{p}=0.043)$ and One-leg scales $(\mathrm{p}=0.043)$ showed significant differences between pre and post intervention. Conclusion: Proprioceptive training with virtual reality proved to be more efficient than the conventional proprioceptive training in elderly subjects in relation to balance, flexibility, mobility and falls.

\section{INTRODUÇÃO}

O aumento da população senescente no Brasil faz propor a discussão sobre eventos incapacitantes que atingem essa faixa etária, dentre as quais se destacam a queda e suas consequências. ${ }^{1,2}$ Esta questão é preocupante, pois constitui um dos principais problemas clínicos e de saúde pública, devido a sua alta incidência, aos elevados custos assistenciais e às diversas complicações como fraturas, lacerações, declive funcional, elevada recorrência de quedas, hospitalizações e morte..$^{3,4}$

Um dos componentes intrínsecos do idoso que o predispõe a quedas é a diminuição do equilíbrio ou do controle postural. ${ }^{2,5,6}$ Este requer manutenção da gravidade sobre a base de sustentação durante situações estáticas e dinâmicas, sendo que o corpo deve ser capaz de responder às translações do centro de gravidade imposta voluntária ou inesperada, como em um tropeço. ${ }^{6,7}$

Uma possibilidade terapêutica de recurso não convencional de reabilitação que vem sendo empregada atualmente na fisioterapia é a realidade virtual. ${ }^{8,9}$ Segundo Albuquerque, ${ }^{10}$ a realidade virtual é uma interação de imagens gráficas, na qual há interface entre o indivíduo e a máquina, ligando os componentes computacionais aos canais sensórios motores, fazendo com que haja simulação de um ambiente real. A exploração de aplicações compostas por cenas e situações simuladas em computadores faz com que o indivíduo acredite estar em outra realidade. Permite associar comportamentos e reações aos objetos virtuais, integrando o usuário ao ambiente virtual - isto é, permite ao indivíduo retratar e interagir com situações imaginárias, envolvendo objetos virtuais estáticos ou em movimentos. ${ }^{10-12}$ Os efeitos gerados por esses ambientes estimulam as mudanças no cérebro, essenciais para o processo de reabilitação. ${ }^{8,10,11}$

A introdução dos jogos virtuais associada à reabilitação, evitando a monotonia do alongamento típico e movimento repetitivo, recebeu o nome de Exergames, uma combinação de games desenvolvidos para atividades físicas, ou seja, jogos que utilizam dispositivos de interação física com o usuário como forma de exercício. É o caso do videogame da Nintendo ${ }^{\circledR}$ Wii e da Balance Board. ${ }^{8,13}$

O controle revolucionário do videogame transforma movimentos reais em comandos na tela, reproduzindo no televisor os movimentos do usuário ("espelho virtual”); os exercícios ajudam as células nervosas a enviar mensagens aos membros, permitindo manter o equilíbrio. ${ }^{14}$ Os jogos exigem percepção visual, coordenação e movimentos sequenciais, razão pela qual foram bem aceitos e com bons resultados como ferramenta coadjuvante no tratamento de pacientes com disfunções neurológicas e lesões musculoesqueléticas, além da correção do equilíbrio, da postura, melhoria da locomoção, da funcionalidade de membros superiores e inferiores, promovendo, ainda, motivação para o paciente. $^{8,9,13,15}$

Os exercícios promovidos na Balance Board enfatizam o controle do movimento porque, durante a realização do treinamento, 
a plataforma capta a instabilidade dos movimentos e proporciona situações às articulações que fisiologicamente ativam impulsos proprioceptivos que são integrados em vários centros sensoriomotores, para regular automaticamente os ajustes na contração dos músculos posturais, mantendo o equilíbrio. ${ }^{8,13}$

Esta pesquisa se justifica pelo uso de uma nova ferramenta em Fisioterapia como forma de intervenção e redução das quedas em idosos. O objetivo do estudo foi avaliar os efeitos de um programa de treinamento fisioterapêutico convencional e de um protocolo com a utilização do videogame associado à plataforma Balance Board em indivíduos idosos, em relação ao equilíbrio, mobilidade, flexibilidade e quedas.

\section{MATERIAIS E MÉTODO}

Trata-se de pesquisa quase-experimental, desenvolvida nas Clínicas Integradas da Faculdade Evangélica do Paraná (FEPAR), na cidade de Curitiba-PR, no período de agosto a outubro de 2010. O projeto foi aprovado pelo Comitê de Ética em Pesquisa da Sociedade Evangélica Beneficente de Curitiba, sob o protocolo $\mathrm{n}^{\circ}$ 3.751/10, de acordo com as normas e diretrizes da resolução CNS no 196/96 e suas complementares.

A amostra foi constituída por 32 indivíduos, com idade entre 60 e 80 anos, participantes da Faculdade Aberta à Terceira Idade (FATI) da FEPAR, dividido em dois grupos - um grupo controle (GC) e um grupo experimental (GE) selecionados aleatoriamente, de forma voluntária, devidamente esclarecidos e orientados quanto à natureza e ao significado do estudo proposto por meio do Termo de Consentimento Livre e Esclarecido (TCLE). Os critérios de inclusão foram idosos de ambos os sexos, hígidos, com marcha independente. Os critérios de exclusão foram: idosos com alterações neurológicas, cardiorrespiratória ou outras disfunções que impedissem a marcha independente.

Para a triagem da amostra, foi realizada uma entrevista inicial para coleta de dados pessoais (idade, sexo, etnia) e uma avaliação funcional, sendo aplicados testes individualmente para comparação e análise dos efeitos do programa proposto. A avaliação foi realizada pelo mesmo examinador, seguindo os critérios de inclusão em idosos hígidos e marcha independente, e exclusão em idosos não hígidos, delimitando assim a amostra do estudo.

A avaliação funcional foi realizada por meio dos seguintes testes:

- Escala de Equilíbrio de Berg: desenvolvida e validada por Berg et al. e adaptado para sua aplicação no Brasil. ${ }^{16}$ Esta escala avalia o desempenho do equilíbrio funcional em 14 testes como a habilidade de sentar, ficar de pé, alcançar, girar em volta de si mesmo, olhar por cima de seus ombros, ficar sobre apoio unipodal, e transpor degraus. A pontuação total é de 56 pontos sendo que índice igual ou menor a 36 está associado a $100 \%$ de risco de quedas. ${ }^{17,18}$

- Teste de Alcance Funcional ou Functional Reach Test (FRT) - TAF: desenvolvido por Duncan et al. ${ }^{19}$ para avaliar a capacidade do idoso de mover-se voluntariamente até os limites de estabilidade anterior e lateral, ou seja, seu limite de alcance funcional., ${ }^{6,17}$

- Avaliação da Marcha e Equilíbrio Orientada pelo Desempenho ou Performance Oriented Mobility Assessment (POMA): criado por Tinetti, ${ }^{20}$ é um dos testes mais utilizados com o objetivo de verificar os padrões de mobilidade e determinar a possibilidade de quedas em idosos. Esse instrumento avalia o equilíbrio do idoso em diversas posições, além de avaliar a marcha. ${ }^{16,20}$

- Escala Unipodal - Unipedal Stance: tem como objetivo avaliar o desempenho de quanto o idoso consegue permanecer em apoio unipodal em diversas condições sensoriais. $^{20}$

Para avaliar as quedas, foi utilizada a Escala Internacional de Eficácia de Quedas (FES-I), 
reelaborada pela rede europeia de prevenção às quedas (PRoFaNE - Prevention of Falls Network Europe). A FES-I apresenta questões sobre a preocupação com a possibilidade de cair ao realizar 16 atividades, com respectivos escores de 1 a 4. O escore total pode variar de 16 (ausência de preocupação) a 64 (preocupação extrema). ${ }^{1}$

Os indivíduos foram separados aleatoriamente em dois grupos:

- Grupo 1: grupo controle (GC) com treinamento proprioceptivo sem modificação.

- Grupo 2: grupo experimental (GE) com treinamento proprioceptivo modificado (olhos vedados) e utilização da ferramenta tecnológica.

Os atendimentos ocorreram no período vespertino, no horário das $13 \mathrm{~h} 30 \mathrm{~min}$ às 15 horas, duas vezes por semana (terças e quintas-feiras), totalizando dez atendimentos com duração de 30 minutos nos dois grupos. No início do treinamento, ambos os grupos realizaram aquecimento durante dez minutos, sendo em seguida separados em GC e GE para a realização das atividades propostas.

O treinamento proprioceptivo para o GC baseava-se em um circuito composto de: 1) balancinho com apoio inicial bipodal e após bipodal; 2) prancha freeman redonda com equilíbrio em duas pranchas simultaneamente e apoio unipodal em cada prancha; 3) prancha freeman quadrada com apoio inicial bipodal e após bipodal; 4) cama elástica individual com pliometria inicial bipodal e após unipodal; 5) skate $(30 \mathrm{~cm}, 60 \mathrm{~cm}$ e $90 \mathrm{~cm}$ de comprimento) em posição de ortotastimo, com apoio unipodal e efetuando cadeia isocinética fechada de flexão e extensão de quadril e membro inferior; 6) disco twist com apoio unipodal em dois discos individuais simultaneamente, realizando cadeia isocinética fechada de extensão em membros inferiores e rotação de tronco e quadril.
O treinamento proprioceptivo para o GE consistia de um percurso retangular de $3,00 \times 2,00 \mathrm{~m}$, por onde os indivíduos passavam duas vezes com os olhos vedados orientado e auxiliado pelo terapeuta. $O$ percurso era composto de obstáculos dispostos no solo e na barra paralela; o primeiro consistia de blocos de E.V.A. de $1 \mathrm{~cm}$ de altura por $32 \times 32 \mathrm{~cm}$ (total de 15 blocos de E.V.A.); um rolo de espuma densidade macia $102 \times 18 \mathrm{~cm}$; um rolo de espuma densidade dura $102 \times 18 \mathrm{~cm}$; um rolo de espuma densidade dura $102 \times 25 \mathrm{~cm}$; sete bastões $134 \times 2 \mathrm{~cm}$; um travesseiro de lona $66 \times 49 \mathrm{~cm}$; duas cunhas de espuma densidade média $54 \times 51 \mathrm{~cm}$; uma sacola de pano com plásticos recortados em tiras $42 \times 48 \mathrm{~cm}$; dois colchonetes $148 \times 53 \mathrm{~cm}$; travesseiro não alérgico de algodão $30 \times 40 \mathrm{~cm}$. $\mathrm{Na}$ barra paralela, de aproximadamente $3,00 \mathrm{~m}$, constavam os seguintes objetos: dois sacos de bolas de gudes com 50 bolinhas cada; um tapete antiderrapante de silicone $46 \times 50 \mathrm{~cm}$; um saco de gel gelado $26 \times 15 \mathrm{~cm}$; um saco com areia $41 \times 30 \mathrm{~cm}$; um tapete áspero $71 \times 40 \mathrm{~cm}$, sendo a distância entre cada objeto de $30 \mathrm{~cm}$.

Para a utilização da ferramenta tecnológica, foi utilizada a console $W i i$ da Nintendo associada ao televisor de 20 polegadas, a Balance Board e quatro jogos selecionados do Wii Fit Plus:

1. Obstacle Course: o personagem passa por vários obstáculos em quatro fases; tem como objetivo passar pelos obstáculos num tempo predeterminado.

2. Perfect 10: o personagem desloca-se para as laterais, para trás e para frente, conforme a numeração referida na tela. Objetivo do jogo: maior pontuação pela velocidade do movimento juntamente com a numeração indicada.

3. Soocer Keading: o principal personagem é um goleiro que fica no meio da trave e tem como objetivo cabecear o máximo de bolas possíveis e desviar de outros objetos que são lançados, como chuteira e brinquedos de pelúcia. 
4. Cross Dresser: o personagem atravessa uma corda em pé andando, que está amarrada entre dois edifícios e desvia-se de uma máquina que segue em sua direção. Tem como objetivo manter o equilíbrio em todo o percurso.

Para a realização dos exercícios, o indivíduo idoso permanecia na posição ortostática, pés descalços ou com sapato antiderrapante, sendo que antes da realização do procedimento o terapeuta demonstrava a maneira correta de realizar os exercícios.

Para a análise estatística, foram utilizados os testes não paramétricos de diferenças entre médias pareadas (Wilcoxon pareado) para comparar cinco variáveis (Escala de Berg; Avaliação da Marcha e Equilíbrio Orientada pelo Desempenho (POMA); Escala Unipodal; Teste de Alcance Funcional Anterior e o Teste de Alcance Funcional Lateral, em momentos pré e pós-intervenção em cada um dos dois grupos estudados (GE e GC).

As análises estatísticas e de gráficos foram efetuadas com o pacote estatístico Statistica (STATSOFT). ${ }^{21}$ Foram encontradas diferenças entre o momento pré e pós-intervenção nos dois grupos (GE e GC), e um novo teste foi aplicado para avaliar se houve melhora maior em um dos dois grupos. Para tanto, as diferenças entre o momento pré e pós foram calculadas por meio da subtração do valor final (pós) menos o valor inicial (pré). O teste não paramétrico de Mann-Whitney foi usado para a comparação dos dois grupos. ${ }^{21}$

\section{RESULTADOS}

A amostra total foi constituída por32indivíduos idosos de ambos os sexos, aleatoriamente divididos em 16 no GE (idade $=66,88$ anos) e 16 no GC (idade $=67,63$ anos).

$\mathrm{Na}$ análise da Escala Internacional de Eficácia de Quedas (FES-I), os resultados das médias encontradas no GE foram 19,63 pontos $\pm 3,02$ pontos e no GC média de 21,88 pontos $\pm 5,77$ pontos, não havendo diferenças significativas em relação ao medo de cair. Nesta amostra, o histórico de queda do GE foi em média de 0,38 $\pm 0,74$ e do GC a média foi de $0,38 \pm 0,52$.

Para o GE, somente a variável Escala de Berg não apresentou diferença significativa $(\mathrm{p}<0,05)$ entre os momentos pré e pós-intervenção (tabela 1). Apesar de algumas variáveis se apresentarem com valores médios diferentes, esta variação não é significativa - não é possível definir se tal variação se deu por uma mudança real da variável ou pelo simples acaso.

Tabela 1 - Valores de média \pm desvio-padrão das cinco variáveis medidas nos momentos pré e pósintervenção do GE (n=16). Curitiba, PR, 2010.

\begin{tabular}{lccc}
\hline \multicolumn{1}{c}{ Variável } & Pré & Pós & p \\
\hline Berg & $52,75 \pm 4,37$ & $55,50 \pm 1,07$ & 0,068 \\
Escala Unipodal & $39,38 \pm 26,40$ & $50,63 \pm 27,50$ & $\mathbf{0 , 0 1 8 ^ { * }}$ \\
POMA & $53,63 \pm 3,25$ & $56,13 \pm 1,46$ & $\mathbf{0 , 0 1 8 ^ { * }}$ \\
Alcance Funcional Anterior & $94,88 \pm 17,45$ & $109,88 \pm 19,74$ & $\mathbf{0 , 0 1 2 ^ { * }}$ \\
Alcance Funcional Lateral & $59,25 \pm 7,91$ & $72,38 \pm 13,34$ & $\mathbf{0 , 0 1 2 ^ { * }}$ \\
\hline
\end{tabular}

${ }^{*} \mathrm{p}<0,05$. Os valores de $\mathrm{p}$ indicados referem-se ao teste de Wilcoxon pareado. 
A variável "equilíbrio" em diversas condições sensoriais, avaliada por meio da Escala Unipodal, apresentou nos dois grupos (GE e GC) diferenças significativas. Esta variável, avaliada por meio da Escala de Berg nos dois grupos, não apresentou diferenças significativas entre os momentos pré e póstratamento no GE.

Tabela 2 - Valores de média \pm desvio-padrão das cinco variáveis medidas nos momentos pré e pósintervenção do GC (n=16). Curitiba, PR, 2010.

\begin{tabular}{lccc}
\hline \multicolumn{1}{c}{ Variável } & Pré & Pós & p \\
\hline Berg & $52,00 \pm 5,86$ & $54,75 \pm 1,28$ & 0,178 \\
Escala Unipodal & $16,50 \pm 11,35$ & $23,63 \pm 13,77$ & $\mathbf{0 , 0 4 3}$ \\
POMA & $54,13 \pm 4,55$ & $56,50 \pm 0,76$ & $\mathbf{0 , 0 4 3}$ \\
Alcance Funcional Anterior & $33,48 \pm 5,83$ & $38,09 \pm 4,76$ & 0,091 \\
Alcance Funcional Lateral & $19,44 \pm 5,81$ & $21,75 \pm 5,91$ & 0,401 \\
\hline
\end{tabular}

$*_{\mathrm{p}}<0,05$. Os valores de $\mathrm{p}$ indicados referem-se ao teste de Wilcoxon pareado.

A tabela 2 mostra as médias entre o GE e GC, apresentando melhora significativa no GE. Já para o GC, tanto POMA como a Escala
Unipodal apresentaram diferenças significativas entre o momento pré e pós-intervenção.

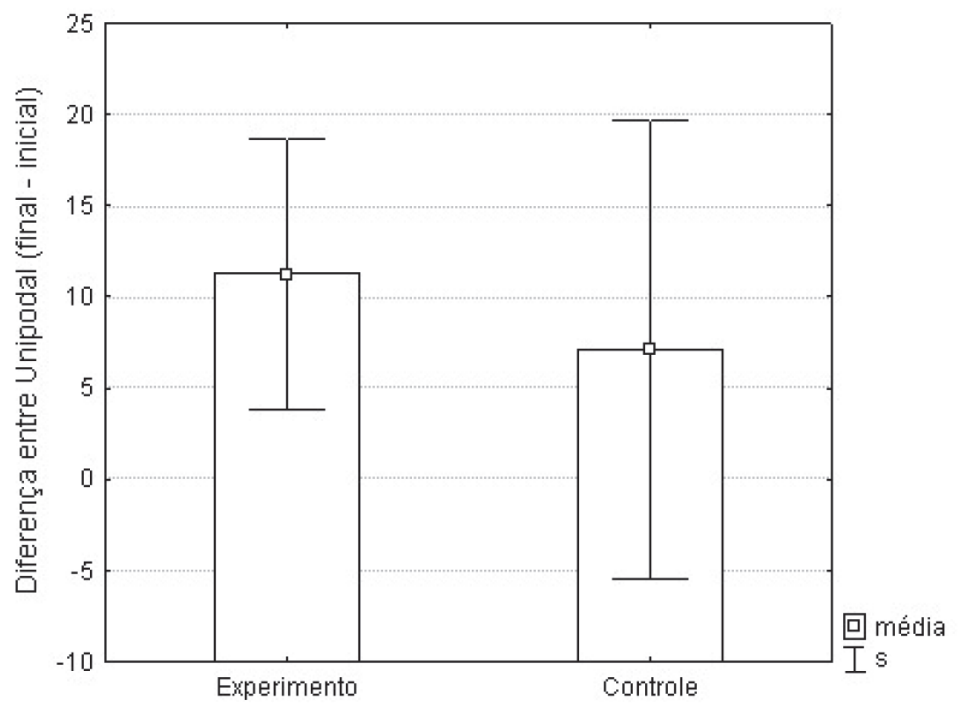

Figura 1 - Médias e desvios-padrões das diferenças da Escala Unipodal entre os dois grupos estudados. Curitiba, PR, 2010.

Na figura 1, observa-se diferença significativa no GE após dez atendimentos em relação à Escala Unipodal. 


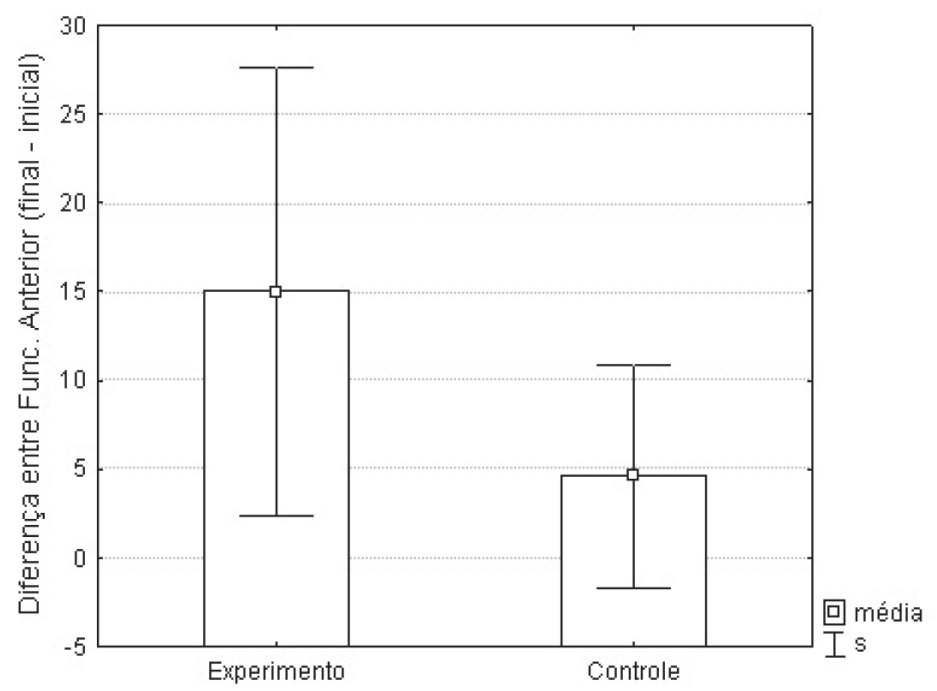

Figura 2 - Médias e desvios-padrões das diferenças do Alcance Funcional Anterior entre os dois grupos estudados. Curitiba, PR, 2010.

$\mathrm{Na}$ figura 2 observa-se uma diferença significativa no GE após 10 atendimentos em relação à flexibilidade lateral.

\section{DISCUSSÃO}

A utilização de tecnologias de Exergame é possibilidade recente de fácil aplicabilidade, baixo custo, de aspecto lúdico que exerce no usuário fascínio pelo ambiente virtual, além de proporcionar habilidades sensoriais e motoras devido ao estímulo perceptivo e de atuação concedidas por meio de mecanismos de realidade virtual. ${ }^{8,13}$

De acordo com Carvalho et al., ${ }^{22} \mathrm{em}$ pesquisa realizada com idosos, obteve-se diminuição dos graus de oscilação com o treinamento proprioceptivo. Esses autores ressaltam que a propriocepção e a informação sensorial são fatores importantes para a manutenção do equilíbrio postural e que o treinamento proprioceptivo aumenta esses estímulos. Ergen \& Ulkar ${ }^{23}$ relatam que os programas de treinamento proprioceptivo devem ser uma prática regular, continuada, privilegiandose a qualidade mais do que a quantidade. Os exercícios devem ser graduais, quer em termos de intensidade, quer de complexidade, realizados em várias superfícies de apoio, com olhos abertos e fechados; devem incluir exercícios que melhorem o sentido e a consciência de movimento articular, que realcem a estabilidade articular dinâmica e o controle neuromuscular reativo, de fácil compressão e desempenho.

A diferença entre $\mathrm{O}$ treinamento proprioceptivo realizado no GC e no GE, aplicados nesta pesquisa, é a forma como são realizados os exercícios. No primeiro caso, os inputs proprioceptivos são gerados por via eferente, oferecidos pelos equipamentos por meio de treinamento ativo dinâmico. No GE, treinamento na Balance Board, o estímulo ocorre por via aferente, ou seja, o paciente visualiza a ação no televisor e faz com que, por meio da interação com o jogo, ocorram oscilações que ativem inputs proprioceptivos em cima da Balance Board de forma estática. Ambos os treinamentos têm a finalidade de aumentar e/ou restabelecer a autoconfiança do idoso em relação a quedas, por meio de um novo engrama sensorial (memória).

Em relação às variáveis POMA, Unipodal, Alcance Funcional anterior e lateral, houve 
melhora significativa no GE em comparação ao GC, resultado que pode estar relacionado à interação do indivíduo idoso com o ambiente virtual. Este aspecto é um dos mais importantes da interface tecnológica e está relacionado com a capacidade do console Nintendo ${ }^{\circledR}$ Wii e da Balance Board em detectar as ações do paciente e reagir instantaneamente. ${ }^{8,13} \mathrm{O}$ esforço para executar bem as jogadas pode provocar impactos positivos no organismo, como o fortalecimento da musculatura, facilidade para recuperação dos movimentos, estímulo da atividade cerebral e aumento da capacidade de concentração e equilíbrio. Necessita, porém, que as atividades de reabilitação sejam supervisionadas por um especialista, como um fisioterapeuta, para auxiliar durante a utilização do recurso, a fim de aperfeiçoar o tratamento e prevenir lesões e quedas..$^{8,10,11,14}$

Em relação às variáveis "flexibilidade anterior elateral" (FRT), observa-se diferença significativa no GE pré e pós-teste. Consta na literatura que a diminuição da flexibilidade afeta o equilíbrio postural e a diminuição do equilíbrio não só provoca deterioração dos movimentos, mas também aumenta o risco de quedas. ${ }^{4}$ Lopes et al. ${ }^{24}$ relatam que o FRT é um teste cuja execução exige alteração na configuração postural, o que promove instabilidade por meio da projeção do corpo para frente e para os lados, simulando uma atividade de alcance. A inabilidade ou incapacidade para execução sugere presença de déficit de equilíbrio, comprometendo a estabilidade, sendo um indicativo para que as quedas ocorram. Neste estudo, pode-se correlacionar o FRT com os escores baixo do FES-I, corroborando Figueiredo et al., ${ }^{19}$ que destacam o aumento da idade como um dos fatores de risco para a manifestação do medo de cair, indicativo de declínio de reserva funcional, decorrente do processo de envelhecimento, fazendo da queda um evento potencialmente grave. A percepção dessas perdas por parte dos idosos pode gerar um sentimento de baixa confiança e medo de cair. ${ }^{24}$

É consenso na literatura que quanto maior o número de riscos presentes, maior a chance de queda. Os principais fatores determinantes de quedas apontados na maioria dos estudos foram idade avançada; sexo feminino; presença de declínio cognitivo; limites de mobilidade, como distúrbios de marcha, equilíbrio corporal e fraqueza muscular; de inatividade; histórico de quedas anteriores e de fraturas; comprometimento na capacidade de realizar atividades de vida diária e uso de medicações psicotrópicas, assim como uso de várias medicações concomitantes. ${ }^{3,18,25-28}$

Segundo Souza, ${ }^{29}$ durante o envelhecimento, ocorre perda de propriocepção articular. Esse decréscimo é apontado na literatura como fator de risco de quedas, assim como problemas somatossensoriais, sensoriomotores e vestibulares. ${ }^{30,31}$

Alfieri et al. ${ }^{4}$ relatam que, por depender dos sistemas somatossensorial, vestibular e visual, o controle postural no idoso está alterado. No presente estudo, foi observado que na variável Unipodal e POMA, em que esses sistemas eram postos à prova, observou-se que no GE houve melhora significativa comparada ao GC no pósteste. De acordo com Cruz et al., ${ }^{5}$ esta melhora se justifica pela contribuição visual na manutenção do equilíbrio e tem explicação na tendência da força de acoplamento entre informação visual e oscilação postural.

De acordo com pesquisas realizadas, a Balance Board pode ser adaptada às limitações do paciente, pelo fato de o aparelho utilizar acelerômetros para mensurar os movimentos realizados em três dimensões, ampliando os aspectos da interação humana e imersão em um game. ${ }^{8,13,32}$ Reitera-se, nesses estudos, o aspecto lúdico que influencia na motivação para o game, incentivando o usuário para as atividades físicas. ${ }^{8,9,13}$

A motivação e adesão ao tratamento no GE aumentaram quando o game foi utilizado por mais de um indivíduo ou com a presença de outros participantes no ambiente. Promoveram também a interação social e a competitividade, no sentido de realizar os movimentos necessários para conseguir maior pontuação, corroborando os achados de Vaghetti \& Botelho. ${ }^{13}$ Esses autores 
analisaram o potencial da nova tecnologia, comparando os efeitos dos games tradicionais e do Exergame, e concluíram que este aumenta o nível de atividade física, permitindo ao usuário maior gasto calórico e aumento da frequência cardíaca durante o exercício, que são aspectos fundamentais para a promoção da saúde e o combate à obesidade. Sugere-se também a inclusão da ferramenta para tratamento do equilíbrio, controle postural, reabilitação de segmentos corporais e treinamento de cirurgiões. ${ }^{13}$

No estudo de Schiavinato et al., ${ }^{11}$ foi utilizado um protocolo com exercícios de equilíbrio do Wii Fit com a Balance Board, no qual se verificou melhora do equilíbrio. Isto sugere que a nova ferramenta tecnológica tem a vantagem de ser um equipamento estável, prático e de boa relação custo $\mathrm{x}$ benefício que permite trabalhar equilíbrio sem risco de queda, quando bem orientado e auxiliado pelo terapeuta, corroborando achados de outros autores..$^{8,13-15}$

Analisando os efeitos nos dois grupos, observou-se que o GE tratado com o treinamento proprioceptivo, associado à nova ferramenta tecnológica, apresentou mais variáveis positivas ao indivíduo idoso quando comparado ao GC nesta amostra. Ou seja, o novo recurso

\section{REFERÊNCIAS}

1. Camargos FFO, Dias RC, Dias JMD, Freire MTF. Adaptação transcultural e avaliação das propriedades psicométricas da Falls Efficacy Scale International em idosos brasileiros. Rev Bras Fisioter 2010;14(3):237-43.

2. Meireles AE, Pereira LMS, Oliveira TG, Christofoletti G, Fonseca AL. Alterações neurológicas fisiológicas ao envelhecimento afetam o sistema mantenedor do equilíbrio. Rev Neurocienc 2010;18(1):103-8.

3. Pimentel RM, Scheicher ME. Comparação do risco de queda em idosos sedentários e ativos por meio da escala de equilíbrio de Berg. Fisioter Pesqui 2009;16(1):6-10.

4. Alfieri FM, Teodori RM, Montebelo MIL. Mobilidade funcional de idosos submetidos a intervenção fisioterapêutica. Saúde Rev Piracicaba 2004;6(14):45-50. tecnológico, quando comparado ao protocolo tradicional de treinamento proprioceptivo, mostrou-se mais eficiente no incremento do equilíbrio, da mobilidade e da flexibilidade, podendo diminuir o risco de quedas.

Cientes dessa realidade, a proposta de um protocolo fisioterapêutico com o uso do videogame Wii associado à plataforma Balance Board nas rotinas semanais de indivíduos idosos poderia auxiliar na melhora do equilíbrio, propriocepção e, consequentemente, na prevenção de quedas de forma mais interativa e lúdica. Relacionando a interface tecnológica e equilíbrio, sugere-se a continuidade desta pesquisa por meio de estudos mais aprofundados do efeito da utilização de videogames em idosos, em relação ao tamanho da amostra, bem como ao número de intervenções do treinamento proprioceptivo.

\section{CONCLUSÃO}

É possível concluir que a nova proposta de realidade virtual aplicada no treinamento proprioceptivo de pacientes idosos em relação ao equilíbrio, mobilidade, flexibilidade e quedas, mostrou ser mais eficiente que o treinamento proprioceptivo convencional, podendo assim, contribuir no processo de reabilitação.
5. Cruz A, Oliveira EM, Melo SIL. Análise biomecânica do equilíbrio do idoso. Acta Ortop Bras 2010;18(2):96-9.

6. Borges FS. Estudo da percepção do idoso institucionalizado em relação ao seu alcance funciona [dissertação de Mestrado]. Brasília: Universidade Católica de Brasília; 2007 [acesso em 10 set 2009]. Disponível em: <http://www. observatorionacionaldoidoso.fiocruz.br/ biblioteca/_monografias/4.pdf>

7. Horak FB. Postural orientation and equilibrium: what do we need to know about neural control of balance to prevent falls? Age Ageing 2006;35(Suppl 2):ii7-11.

8. Sousa FH. O uso do Nintendo® Wii como instrumento de reabilitação na Fisioterapia: revisão bibliográfica [acesso em 6 out 2010]. Disponível em: http://artigocientifico.tebas.kinghost.net/uploads/ artc_1283750849_64.pdf 
9. Dias RS, Sampaio ILA, Taddeo LS. Fisioterapia X Wii: a introdução do lúdico no processo de reabilitação de pacientes em tratamento fisioterápico. In: VIII Brazilian Symposium on Games and Digital Entertainment; 2009 Oct 8-10; Rio de Janeiro; 2009.

10. Albuquerque EC, Scalabrin EE. O uso do computador em programas de reabilitação neuropsicológica. Psicol Argum 2007;25(50):269-75.

11. Schiavinato AM, Baldan C, Melatto L, Lima LS. Influência do Wii Fit no equilíbrio de paciente com disfunção cerebelar: estudo de caso. J Health Sci Inst 2010;28(1):50-2.

12. Ferreira E. Paradigmas do jogar: interação, corpo e imersão nos videogames. In: VIII Simpósio Brasileiro de Jogos e Entretenimento Digital. Rio de Janeiro: Pontifícia Universidade Católica; 2009.

13. Vaghetti CAO, Botelho SSC. Ambientes virtuais de aprendizagem na educação física: uma revisão sobre a utilização de Exergames. Ciênc Cognição 2010;15(1):76-88.

14. Halton J. Virtual rehabilitation with video-games: A new frontier for occupational therapy. J Occupational Therapy Now 2008;9(6):12-4.

15. Olmedo PJ. Videoconsola Wii: Lesiones provocadas por uso inadecuado versus aportaciones al mantenimiento y restauración de la salud. Trances 2010;2(1):1-13.

16. Berg KO, Wood-Dauphinee SL, Williams JI, Maki B. Measuring balance in the elderly: validation of an instrument. Can J Public Health 1992;83(Suppl 2):S7-11.

17. Soares KV, Figueiredo KMOB, Caldas VVA, Guerra RO. Avaliação quanto à utilização e confiabilidade de instrumentos de medida do equilíbrio corporal em idosos. PubICa 2005 [acesso em 12 set 2010];1(2). Disponível em: http://www.reitoria.ufrn.br/ojs/index. $\mathrm{php} /$ publica/article/viewFile/19/12.

18. Menezes RL, Bachion MM. Estudo da presença de fatores de riscos intrínsecos para quedas, em idosos institucionalizados. Ciênc Saúde Coletiva 2008;13(4):1209-18.

19. Figueiredo KMOB, Lima KC, Guerra RO. Instrumentos de avaliação de equilíbrio corporal em idosos. Rev Bras Cineantropom Desempenho Hum 2007;9(4):408-13.

20. Gomes GC. Tradução, adaptação transculturalmente e exame das propriedades de medida da escala "Performance-oriented mobility assessment" (POMA) para uma amostragem de idosos brasileiros institucionalizados. [dissertação de Mestrado]. Campinas: Faculdade de Educação da Universidade Estadual de Campinas; 2003. 115 p.

21. Statistica for Windows. Release 6.0. Tulsa, Oklahoma: Satsoft Inc; c1984-2001.

22. Carvalho SS, Santos JDM, Oliveira MC, Oliveira MA. Análise do equilíbrio dos idosos através da biofotogrametria computadorizada pós treinamento proprioceptivo. In: XIII Encontro Latino de Iniciação Científica, IX Encontro Latino Americano de Pós Graduação e III Encontro Latino de Iniciação Científica Junior; São José dos Campos: Universidade do Vale do Paraíba; 2009.

23. Ergen E, Ulkar B. Proprioception and ankle injuries in soccer. Clin Sports Med 2008;27(1):195-217.

24. Lopes KT, Costa DF, Santos LF, Castro DP, Bastone AC. Prevalência do medo de cair em uma população de idosos da comunidade e sua correlação com mobilidade, equilíbrio dinâmico, risco e histórico de quedas. Rev Bras Fisioter 2009;13(3):223-9.

25. Câmara LC, Santarém JM, Jacob Filho W. Atualização de conhecimentos sobre a prática de exercícios resistidos por indivíduos idosos. Acta Fisiatr 2008;15(4):257-62.

26. Spirduso WW. Dimensões Físicas do Envelhecimento. São Paulo: Manole; 2005.

27. Marinho MS, Silva JF, Pereira LSM, Salmela LFT. Efeitos do Tai Chi Chuan na incidência de quedas, no medo de cair e no equilíbrio em idosos: uma revisão sistemática de ensaios clínicos aleatorizados. Rev Bras Geriatr Gerontol 2007;10(2):243-56

28. Lemos PV. Análise do controle postural de idosas saudáveis. [dissertação de Mestrado]. São Paulo: Universidade de São Paulo; 2009.

29. Souza A. Propriocepção. São Paulo: Editora MEDSI; 2004

30. Speers RA, Kuo AD, Horak FB. Contribuitions of altered sesation and feedback responses to changes in coordination of postural control due to aging. Gait Posture 2002;16(1):20-30.

31. Kauffman TL. Manual de Reabilitação Geriátrica. Rio de Janeiro: Guanabara Koogan; 2001.

32. Silva SLA, Vieira RA, Arantes P, Dias RC. Avaliação de fragilidade, funcionalidade e medo de cair em idosos atendidos em um serviço ambulatorial de geriatria e gerontologia. Fisioter Pesqui 2009;16(2):120-5. 